\section{$\underset{\text { hommes migrations }}{\text { hommes }}$}

\section{Hommes \& migrations}

Revue française de référence sur les dynamiques

migratoires

$1324 \mid 2019$

Religion et discrimination

\title{
Artiste de la diaspora
}

D’ici ou de là-bas, des musiciens assignés?

\section{François Bensignor}

\section{OpenEdition \\ 1 Journals}

Édition électronique

URL : https://journals.openedition.org/hommesmigrations/8611

DOI : 10.4000/hommesmigrations.8611

ISSN : 2262-3353

Éditeur

Musée national de l'histoire de l'immigration

Édition imprimée

Date de publication : 1 janvier 2019

Pagination : 212-218

ISBN : 978-2-919040-44-5

ISSN : $1142-852 X$

Référence électronique

François Bensignor, « Artiste de la diaspora », Hommes \& migrations [En ligne], 1324 | 2019, mis en ligne le 01 janvier 2019, consulté le 06 janvier 2022. URL : http://journals.openedition.org/

hommesmigrations/8611; DOI : https://doi.org/10.4000/hommesmigrations.8611 


\section{Musique}

\section{Artistes de la diaspora}

D'ici ou de là-bas, des musiciens assignés?

\section{Propos recueillis par François Bensignor}

Le 6 novembre 2018, la Cité internationale des arts accueillait une rencontre-débat proposée par le festival Ville des musiques du Monde autour du thème «Artistes de la diaspora, problématique de l'assignation identitaire». Ouvert au public, ce débat réunissait de nombreux artistes, résidents à la Cité internationale des arts et finalistes du Prix des Musiques d'ici (voir $\left.H \& M, n^{\circ} 1321\right)$, et professionnels œuvrant dans le domaine des musiques du monde, producteurs, programmateurs, représentants d'institutions et de médias, membres du jury du Prix des Musiques d'ici. À l'issue de la rencontre, étaient proclamés les noms des trois lauréats de la deuxième édition du Prix des Musiques d'ici.

\section{Une équipe de France africaine?}

Le texte introduisant la thématique la replaçait dans le contexte d'une actualité récente:

«À l'occasion de la victoire de la France lors de la Coupe du Monde de football, Trevor Noah, animateur vedette à la télévision américaine, d'origine sud-africaine, a créé la polémique en France et dans le monde en parlant de la victoire de l'équipe de France comme celle des "bleus africains". Le débat portait essentiellement sur la question ou non de revendiquer une identité plurielle ou "identité relation" selon l'expression d'Édouard Glissant, sans que cela ne soit perçu comme une déclaration de guerre.

«Ce problème, récurrent depuis plusieurs décennies, de l'assignation identitaire, est également présent dans le monde de la musique. Un musicien français d'origine africaine ou nordafricaine qui joue du blues, du rock est facilement étiqueté "musiques du monde": on peut citer le cas du rocker Rachid Taha, récemment disparu, enfermé dans la case "raï" par les médias, ou la polémique provoquée par l'annulation du concert de Black M pour la commémoration de la bataille de verdun et sa réponse musicale avec le titre "Je suis chez moi"...

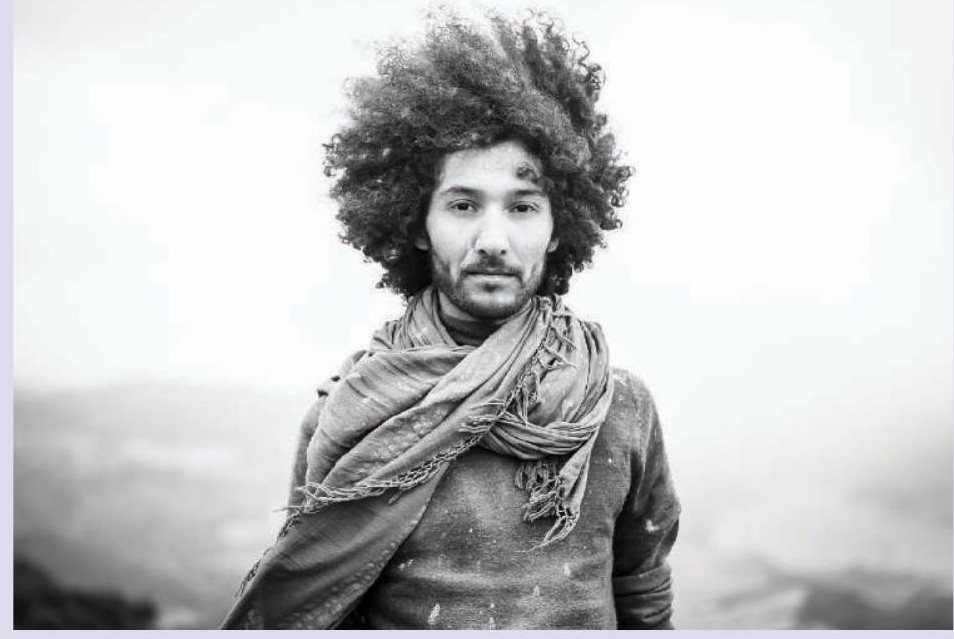

- Walid Ben Selim. (C) Cécile Cellerier.

"Si l'artiste est français d'ascendance étrangère, sa musique est-elle française à part entière ou entièrement à part. Aujourd'hui, les artistes de la nouvelle génération ont décidé de répondre à cette assignation: ils revendiquent cette identité plurielle tout en s'inscrivant dans des courants musicaux majeurs et affirment, par des terminologies propres (afro-punk, afro-rap, électrooriental, etc.), leur singularité.

«Dans l'échange entre Trevor Noah et l'ambassadeur de France à Washington - qui déclarait: "Pour nous il n'y a pas d'identités avec des traits d'union. Les racines sont une réalité individuelle" -, la question de l'assignation identitaire a donc été posée et largement discutée dans les réseaux sociaux.»

Amobé Mévégué, journaliste à TV5Monde et France 24, va s'imposer en aimable et pertinent meneur de jeu, s'adressant à trois intervenants: Lady Apoc, chanteuse togolaise lauréate 2018 du Prix Visa pour la création de l'Institut français, en résidence à la Cité internationale des arts, où elle mène un projet mêlant rock et musique vaudou; Bertrand Dicale, journaliste spécialisé dans la chanson populaire, exerçant notamment sur France Info et France Inter, auteur de nombreux ouvrages, notamment sur Juliette Gréco ou Charles Aznavour, et du récent Ni noire ni blanche, histoires des musiques créoles (Philharmonie de Paris, 2017); Edgar Sekloka, artiste, slameur et écrivain, l'un des trois lauréats du Prix des Musiques d'ici 2018. 
Une question est soumise aux intervenants par Amobé Mévégué: «Ces footballeurs, qui sont d'origine plurielle et appartiennent au corpus basique de la France, deviennent des intermittents de la citoyenneté. Un jour, ils sont vraiment français, l'autre ils ne le sont pas. Ce débat vous a-t-il surpris? Et, selon vous, la question se pose-t-elle?»

Bertrand Dicale affirme ne pas avoir été surpris par la polémique. "Une chose m'a amusée, dit-il. En France, quand on dénonce l'équipe de France comme "africaine", il s'agit d'un discours d'extrême droite. Or Trevor Noah représente l'extrême gauche aux États-Unis... Les termes "Afrique" ou "Africain" sont des armes de jet. J'étais surpris que l'on puisse continuer de se jeter ces mots comme des pavés. En soi, la polémique n'est pas tellement gênante, mais les tournures de style contournées pour aborder ces notions d'Afrique et d'Africain, oui! En tant qu'Antillais descendant d'esclave par mon père, même si je suis plutôt très pâle, je suis un descendant d'Africain. Mais en considérant les millions de chemins de vies différents que l'on peut trouver dans les généalogies de tant de gens, je me demande à partir de quel moment on cesse d'être africain. C'est là que se trouve la question existentielle. » Amobé Mévégué: "Dans la bouche de Trevor Noah, vu de l'Atlantique, tous les Noirs sont africains, Antillais compris. D'ailleurs, l'équipe de France est composée de représentants du Maghreb, des Antilles comme de l'Afrique subsaharienne.»

\section{Rock ou Afrique, faut-il choisir?}

Se tournant vers Lady Apoc, Amobé Mévégué lui demande: «Cette assignation à l'identité, l'avezvous vécue lorsque vous avez fait le grand bond dans la musique en choisissant de jouer du rock et du blues?»

Lady Apoc: "C'est une chose que je vis non seulement au Togo, mais aussi en France. Une sorte de stigmatisation. Aujourd'hui, quand on parle d'identité, on ne parle plus de nature, mais d'origine attachée à la couleur de la peau. À la base, le rock est une musique de fusion, qui a été créée pour briser les frontières raciales. J'ai découvert le rock grâce à mon père, qui en écoutait beaucoup à la maison. J'ai aussi eu la chance de rencontrer le grand artiste togolais Jimi Hope, qui a fait du blues et du rock sa vie. Aujourd'hui, je fais des recherches sur les rythmes vaudous, que je veux associer au rock.

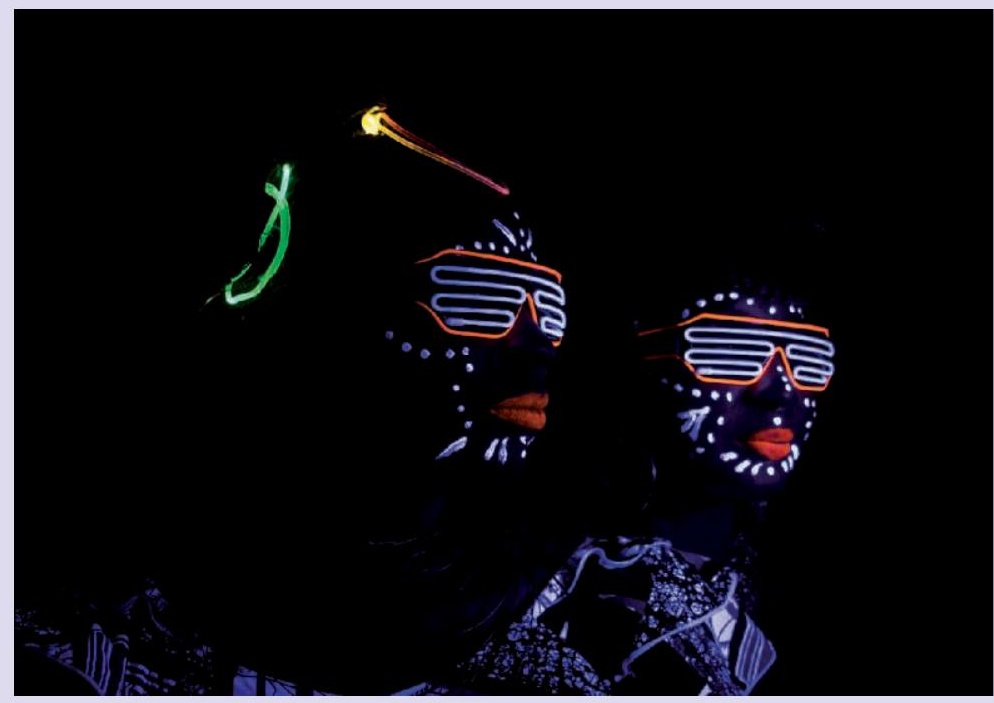

- Germaine Kobo \& Bella Lawson. (c) Roxanne Rinaldo.

« La musique vaudou se joue tout près de chez moi, mais je n'en ai jamais écouté à la maison. Chez moi, j'ai bénéficié d'une culture rock. Le rock est une musique que j'aime profondément et je ne comprends pas pourquoi je devrais me justifier d'avoir choisi cette musique, sous prétexte qu'elle ne ferait pas partie de mon identité ou de ma culture. Je revendique l'identité plurielle que je porte en moi et qui m'a construite. L'identité n'est pas figée, elle évolue dans l'espace, dans le temps, et elle peut devenir encore autre chose. Au Togo, on me dit qu'il faudrait que je joue des rythmes traditionnels comme le "lagbadja". Et ma réponse est: mais pourquoi?

«Lorsque j'arrive en France et que je dis que je fais du rock, on m'explique qu'ici le rock est une communauté, une philosophie de vie dans laquelle je ne vais pas réussir à m'insérer... Alors, je repose la question: mais pourquoi? Et on m'explique que ce n'est déjà pas donné d'en faire quand on est blanc et que, comme je viens d'une culture africaine, faire du rock va paraître ici bien trop bizarre... Que dois-je faire? Jeter tous les acquis qui m'ont construite avec le temps au profit de ce que les gens veulent que je fasse? Non! Alors je continue pour imposer cette musique chez moi et m'insérer dans celle d'ici.»

\section{Et le blues dans tout ça?}

Amobé Mévégué évoque le grand artiste malien Ali Farka Touré, qui jouait la musique traditionnelle 


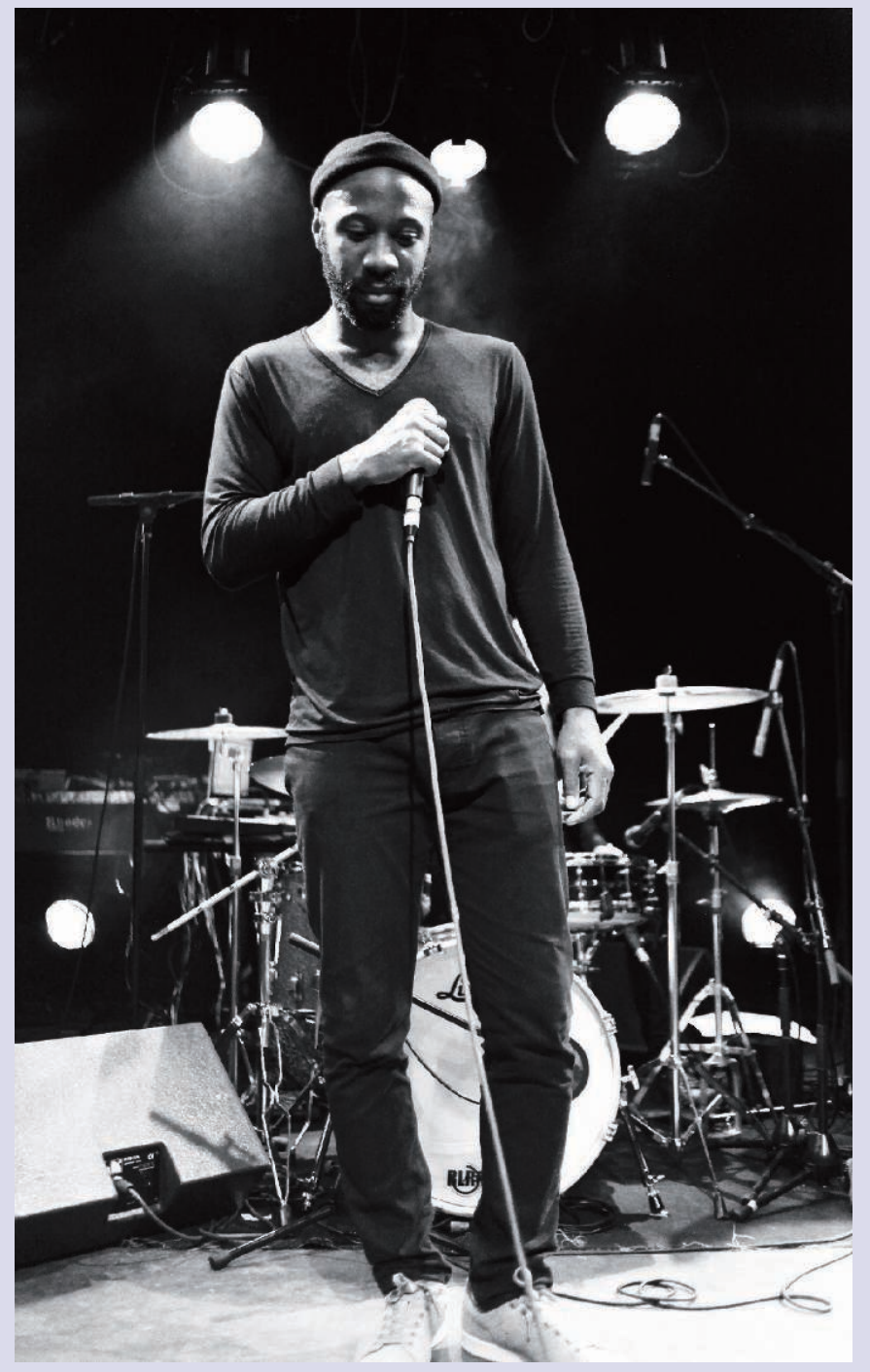

- Edgard Seklola. (C) Kamel Friha.

songhaï de sa région. En l'entendant, des musiciens du delta du Mississippi se dirent que le blues originel devait venir de cette région, car Ali Farka Touré jouait la même musique qu'eux. Ry Cooder, star de la scène américaine, proposa de faire venir Ali Farka Touré pour enregistrer un disque aux États-Unis. Mais Ali Farka Touré lui répondit que, s'il voulait faire cet album, il n'avait qu'à venir luimême dans son village de Niafounké. Et c'est ce qui s'est passé.

Bertrand Dicale rappelle alors une scène du film A Visit to Ali Farka Touré, paru après la mort du grand artiste malien en 2006. Marc Huraux, son réalisateur, à qui l'on doit aussi le beau film Ali Farka Touré, le miel n'est jamais bon dans une seule bouche, paru en 2000, interrogeait le musicien sur sa jeunesse. Celui-ci racontait qu'à 15 ans, il s'était engagé comme assistant chauffeur sur des camions qui parcouraient les routes menant du Mali au Burkina Faso ou en Guinée. Au passage des frontières, qui prenait beaucoup de temps, les camionneurs s'arrêtaient dans les bars-restaurants établis non loin des douanes. C'est là que le jeune Ali Farka Touré découvrit le blues et la voix de John Lee Hooker.

«Bien sûr, il y a de la musique traditionnelle songhaï et peule dans la musique d'Ali Farka Touré, souligne Bertrand Dicale, mais il a aussi découvert le blues à 15 ans, en même temps que les Rolling Stones. À aucun moment dans la mythologie des musiques populaires, on ne dira que le blues vient de Londres ou de Liverpool, parce que de jeunes musiciens anglais y jouent une musique directement inspirée du blues. Mais, parce que Ali Farka Touré est un Africain, on a cette extrême facilité à inverser le jeu des influences: il est naturel qu'un Africain joue du blues...

«On connaît les racines musicologiques du blues. Le blues incorpore des éléments épars d'Afrique de l'Ouest. Mais il naît à la fin du XIXe siècle dans un pays où la traite a cessé en 1807. Quatre générations d'Afro-Américains se sont déjà succédées. Or que reste-t-il dans notre bagage culturel après quatre générations? Il y a quelque chose qui relève de l'assignation identitaire à construire, une sorte d'internationale des musiques noires à laquelle on ne pourrait pas échapper.»

L'artiste musicien Camel Zekri (voir H\&M, $n^{\circ}$ 1285), virtuose de la guitare, répond qu'Ali Farka Touré, à l'origine un joueur de vièle «godjé », n'est pas le seul représentant de la guitare moderne dans sa région. "J'en ai moi-même entendu des centaines, affirme-t-il. Et je veux témoigner de ce que ce blues saharien est une réalité africaine, présente non seulement chez les Songhaï et les Peuls, mais aussi chez les Touaregs, dans la musique gnaoua et les musiques de Mauritanie, c'est-à-dire dans cette vaste région de l'Afrique sahélienne qui a vu fleurir une culture très forte, laquelle s'est transmise aux États-Unis, où elle s'est métissée. Pour moi, il est incontestable que les racines du blues plongent dans ces régions, où les musiques originelles portent d'autres 
noms. Musicalement, le blues relève de formes pentatoniques typiques de ces régions du Sahara et du Sahel.»

\section{Une approche coloniale encore à l'œuvre}

Se tournant vers son troisième intervenant, Amobé Mévégué poursuit: «Vous avez écrit deux romans. Le premier s'intitule Coffee. Ces questions d'assignation identitaire sont-elles prégnantes dans votre culture et dans votre quête?»

Edgar Sekloka: «Je ne pose pas la question comme ça. Je réfléchis d'abord à la façon de me sortir de mes carcans. Le carcan identitaire, je l'ai connu jeune. "Est-ce que je suis Noir parmi les Blancs et Blanc parmi les Noirs, sans pour autant être métisse? Est-ce que je suis métisse, étant franco-camerounais, portant un nom béninois, qui est celui de mon père, que je ne connais pas?" Toutes ces questions figurent dans ma recherche artistique. Ces questionnements me poussent à l'épanouissement.

"Pour moi, "assignation" est un mot que je ne comprends pas. J'ai trouvé belle la réaction de Trevor Noah aux États-Unis. Mais toute la polémique qui a suivi m'a un peu dépassé. Trevor Noah est intervenu depuis les États-Unis, mais c'est l'ambassadeur de France qui a créé la polémique. Et son retentissement en France raconte plutôt le rapport qu'entretient la France avec ses anciennes colonies: il n'est pas question de football, ni d'assignation. Il s'agit juste de cette tendance française à vouloir maintenir sa domination sur ses anciennes colonies.

«Pour moi, un joueur comme Mbappé est un Français dont le nom évoque ses origines. Je vais faire une petite digression en évoquant une dame qui participe aux ateliers d'écriture que j'anime au sein de l'association de solidarité avec les travailleurs immigrés. Elle ne parle pas très bien français. Son père a fait la guerre d'Algérie. Elle a suivi son mari en France et c'était pour elle comme une première blessure. Dans son texte, elle écrit qu'avoir suivi son mari en France a été comme une alliance entre son pays, l'Algérie, et son pays de chute, la France. Pour moi, le cas des joueurs de l'équipe de France relève de cette notion d'alliance. Par eux, s'ils en ont envie, il peut y avoir un meilleur pour les pays dont ils sont issus. Par exemple, de par sa notoriété internationale, Mbappé pourrait permettre à l'équipe du Cameroun de bénéficier d'un meilleur équipement.»

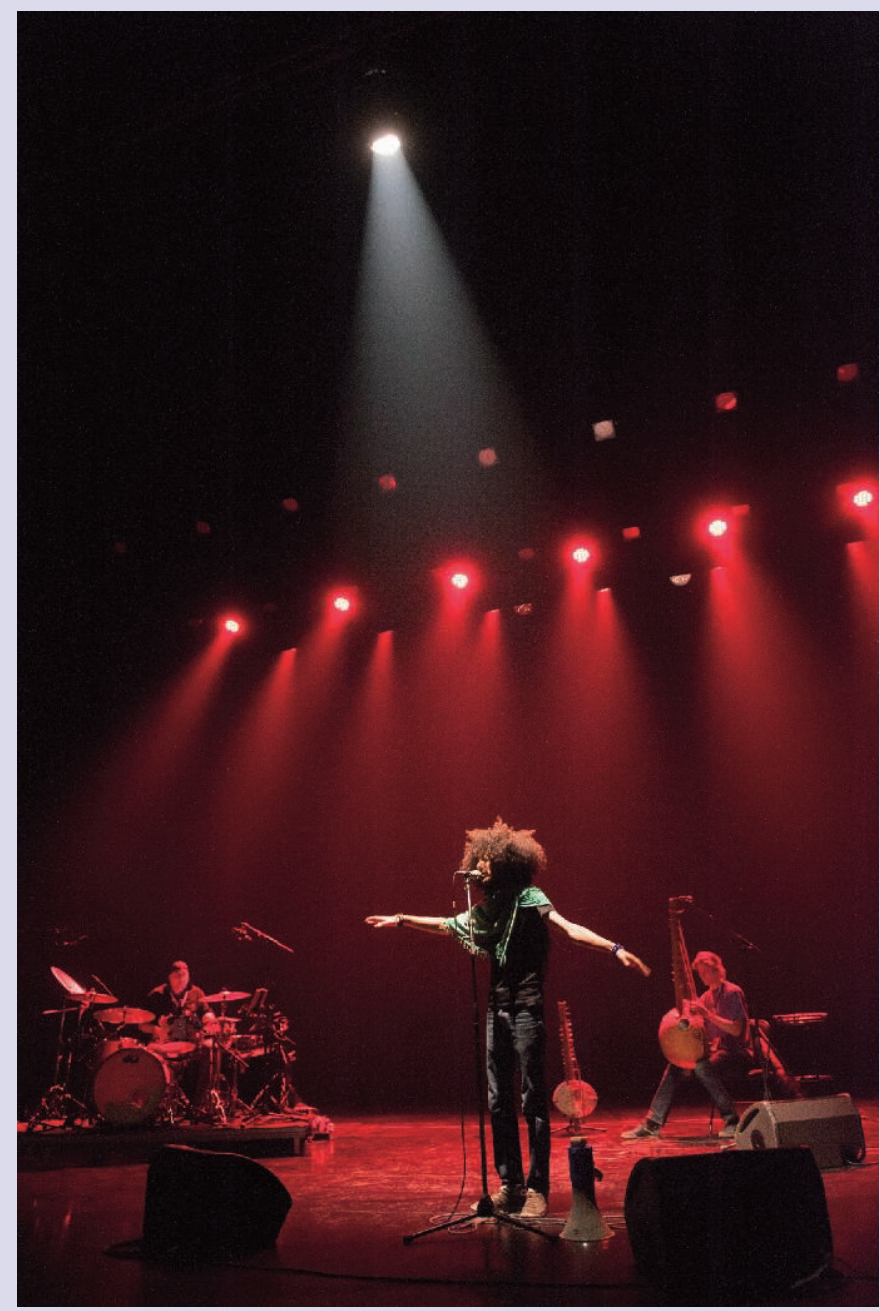

Walid Ben Selim. (C) Cécile Cellerier.

\section{La problématique antillaise}

La chanteuse Ymelda Marie-Louise se saisit du micro qui circule dans la salle. D'origine haïtienne et vivant en Martinique, elle est actuellement en résidence à la Cité internationale des arts.

«Cette problématique dont vous traitez aujourd'hui ne concerne pas que la France et l'Afrique, dit-elle. C'est aussi ce que je vis à la Martinique. En tant que Noire et Haïtienne, j'y rencontre exactement le même problème que les Noirs d'Afrique en France. Je ne vois pas non plus en France une place faite pour les musiques caribéennes au niveau de la diffusion.

"Ce n'est pas une question d'assignation culturelle, mais bien plutôt une méconnaissance de l'autre. Quand on apprend à connaître l'autre, 


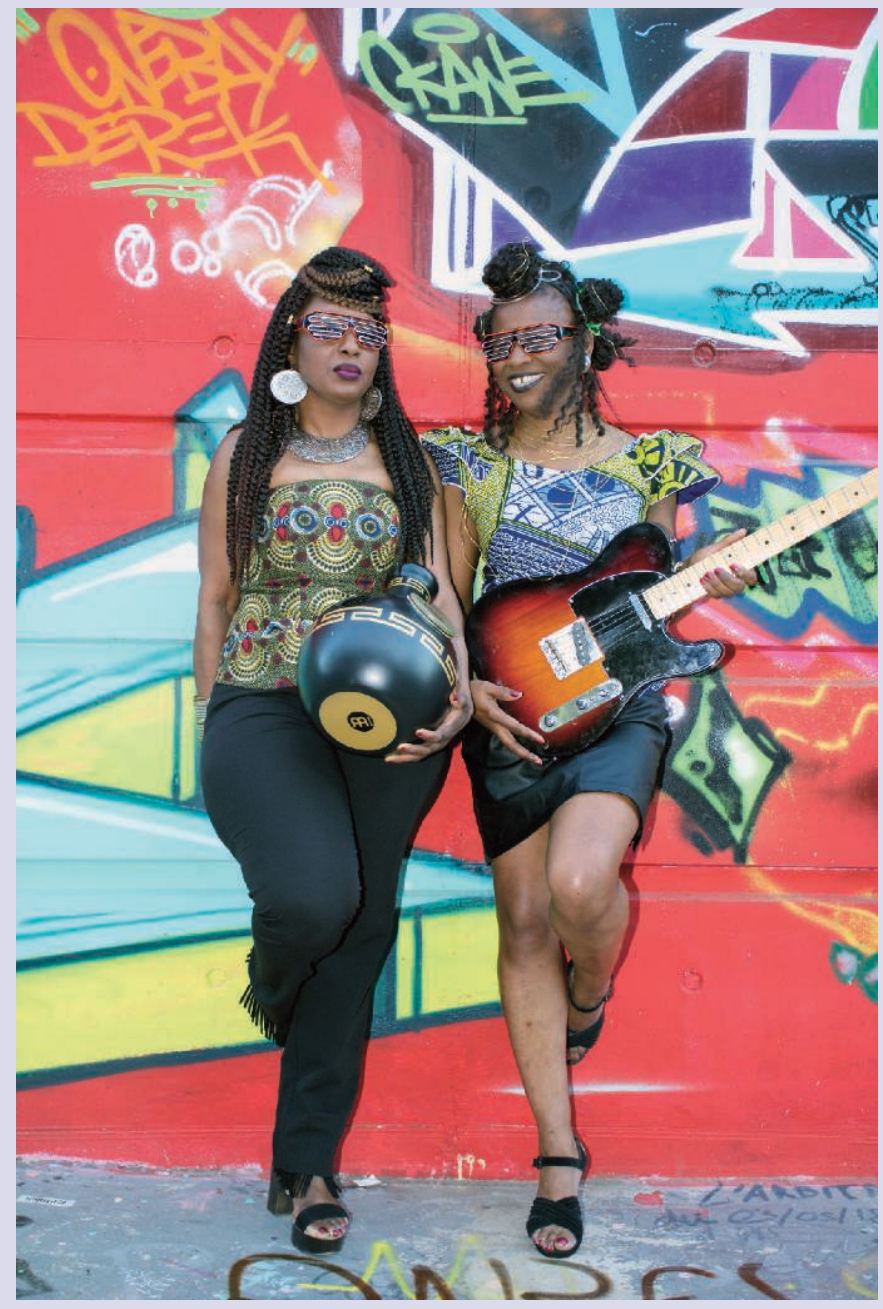

- Germaine Kobo \& Bella Lawson. (c) Roxanne Rinaldo.

on peut obtenir autre chose, plutôt que de rager parce qu'on n'écoute pas la musique qu'on fait. Personnellement, je sais qui je suis. Je viens d'une république. Notre histoire diffère de celle des Martiniquais. Je suis en paix avec moi-même. Je n'ai pas de problème d'assignation culturelle. Je fais la musique qui me correspond tout à fait. Je ne cherche pas à ressembler à l'autre, mais juste à être moi. Mais aussi à faire en sorte que l'on puisse savoir qui je suis et où je vais. Je cherche à vivre avec l'autre et à lui faire comprendre qui je suis. Dès lors que l'on parvient à comprendre qui est l'autre, il n'y a pas de problème pour vivre et avancer ensemble.»

Roger Raspail, maître du gwoka guadeloupéen (musique de tambours, voir H\&M, n 1308 et 1214), implanté en région parisienne, où il a formé et accompagné de nombreux jeunes musiciens, estime qu'il a un vrai problème culturel en France en tant qu'Antillais. "La visibilité de notre musique est vraiment réduite, affirme-t-il, qu'il s'agisse de diffusion ou de médias, et je me demande pourquoi? Est-ce que la France culpabilise? Pourquoi sommes-nous absents?»

Bertrand Dicale répond: "Une des raisons fondamentales pour lesquelles le gwoka n'est pas diffusé en France, c'est que les Français ne comprennent pas le gwoka, parce qu'ils n'arrivent pas à comprendre pourquoi des Français sont africains. Pour eux, des tambours et des nègres, c'est l'Afrique, et pas les Antilles.

"Je me souviens d'un programmateur avec qui j'avais une discussion à ce propos et qui me disait préférer les "djembefola" (maîtres du tambour djembé) de Guinée aux joueurs de gwoka antillais. Or les deux n'ont rien à voir. Le gwoka est une musique très sophistiquée. Par parenthèse, elle est aussi extrêmement gênante pour nous Antillais, parce que nos parents l'ont refusée, qui la taxaient de "mizik a vié nèg" (musique des nègres sales, des vagabonds), et qu'intuitivement on sait bien comment cette musique fonctionne. Aujourd'hui, beaucoup de "tambouyé" (joueurs de tambours ka) vous disent que cette musique est celle de la résistance à l'esclavage, mais pas du tout, c'est une musique de collabos! Une bonne partie des rythmes du gwoka sont des rythmes de travail. Le "graj", le "toumblak" ou le "padjanbèl" sont des rythmes qui accompagnaient la coupe de la canne et celui qui battait tambour à l'époque de l'esclavage était plutôt un contremaître qu'un résistant.

«Et de plus, son choix était très simple: soit il battait le tambour régulièrement et au rythme qui lui était demandé pour que la canne tombe vite, soit on en mettait un autre à sa place et il allait retourner dans le champ. Ce qu'il est douloureux pour nous d'admettre, ce n'est pas d'être des descendants d'esclaves, c'est que l'esclavage se soit perpétué pendant plusieurs siècles...»

Marie-José Justamont, directrice du festival Les Suds à Arles, intervient: "Concernant les musiques antillaises, ne croyez pas qu'elles soient plus discriminées dans les programmations des salles ou des festivals que les musiques des Pays d'Oc, de Corse ou du Massif central... En tant que 
programmatrice, je peux vous garantir que l'on attire plus de monde avec des musiques antillaises qu'avec les musiques de Provence. Le problème est ailleurs. Si l'on considère le jeu "dominant-dominé", il est certain que la culture des provinces se porte mal de ce point de vue.»

\section{Les diasporas ont de la mémoire}

Camel Zékri recentre le débat sur la question des diasporas, populations qui se sont disséminées dans des territoires, où leurs langues d'origine ne sont pas parlées.

«Dans mon cas, dit-il, j'ai hérité de la langue algérienne de mes parents. Mais les gens qui émigrent emmènent dans leurs bagages la culture de l'époque à laquelle ils sont partis. Ainsi, j'ai appris l'arabe que parlaient ma mère et mon père dans leur enfance. Ces expressions de langage, je les porte en moi, et quand je vais en Algérie, il m'arrive d'employer des expressions qui ne sont plus utilisées. Cela signifie que les populations qui se déplacent emportent un patrimoine constitué à une époque donnée et qu'elles le gardent. Il se fige en quelque sorte. De même pour la musique ou l'alimentation.

"Je veux dire que les Noirs qui ont été déportés au Nouveau Monde y sont arrivés avec leurs tambours et leurs musiques. Et ils ont conservé ce patrimoine. Même si la signification de la voix du tambour s'est perdue, il n'empêche que la façon de le jouer et la transmission de cette connaissance ont perduré durant au cours des siècles.

"J'ai fait une recherche sur la musique d'Algérie que pratique ma famille. Les chants traditionnels sont transmis dans une langue qui ne se parle plus, dans la ville dont ma famille est originaire (Biskra). La dernière personne qui connaissait le sens de ces chants était mon grandpère. Cette langue qui est restée dans les chants est devenue pour nous un langage d'onomatopées. Même si l'on connaît ces répertoires et que l'on peut les faire durer pendant des heures, ce sont des mots appris par cœur dont nous avons perdu le sens. Par chance, je suis allé dans un pays voisin, où les gens comprennent encore la langue de nos chants. Ainsi, d'un coup, j'ai vécu cette chose extraordinaire de pouvoir connaitre la signification d'un répertoire que je pensais perdu pour toujours.

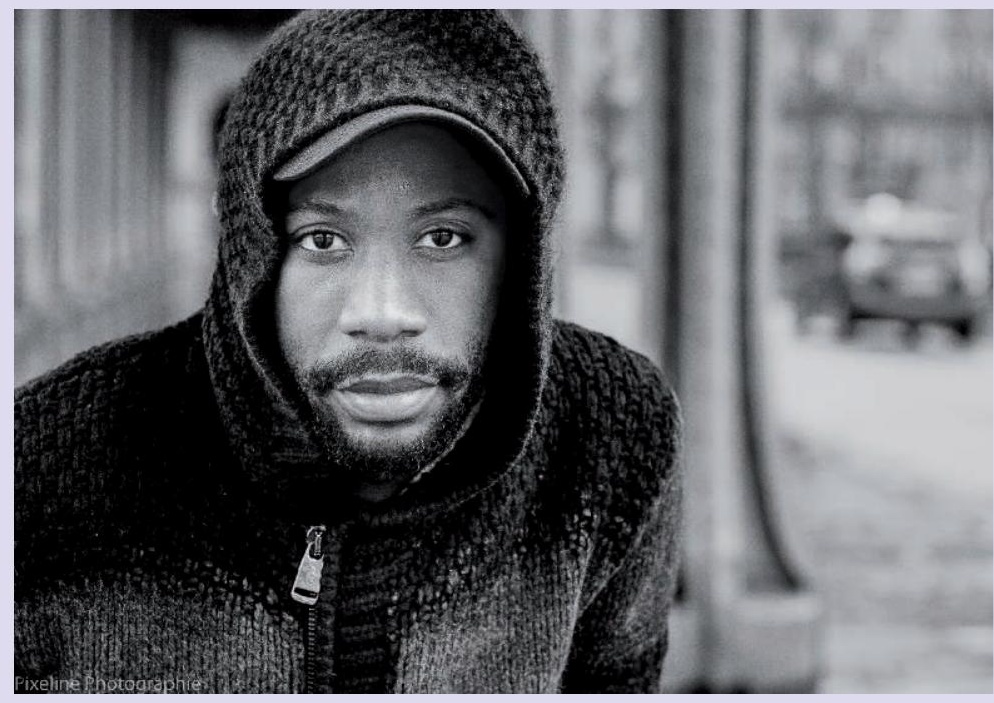

- Edgard Seklola. (c) Pixeline.

«Pour revenir sur cette question de l'enfermement de la culture noire ou africaine, qui est un vrai problème sur lequel il faut travailler, il existe quand même des racines, qui sont portées par réminiscences, par des gestes, que l'on ne soupçonne pas mais qui sont à l'intérieur de nous. Et c'est valable pour le Breton comme pour l'Allemand. Nous sommes porteurs d'éléments culturels qui sont en inconscience et qui peuvent ressortir par une sorte de réminiscence du geste.

"Le geste est le langage le plus fort, qui nous dépasse et qui inscrit les choses dans la pensée. Et si l'on reprend le propos sur les tambours, je réagirais en disant qu'il existe un langage sous-tendu dans le jeu du tambour. Même si les descendants ne connaissent plus la signification de ce langage après quatre générations - ce dont je ne suis pas du tout persuadé-, il reste des clés dans ce jeu de tambours, qui réfèrent directement à ce langage originel.»

\section{Des solutions à rechercher}

En guise de conclusion, Kamel Dafri, le directeur du festival Villes des musiques du Monde invite à déplacer le regard afin de ne pas rester enfermés dans le piège de l'assignation. «Certes, nous devrions relire James Baldwin, revoir I am not your Negro, le magnifique documentaire qui lui est consacré, dit-il. Mais c'est la modeste contribution du Prix des Musiques d'ici que d'essayer de déplacer le regard, d'inviter à réfléchir sur le fait que Khaled 
ou Rachid Taha n'appartiennent pas à un pays fantasmé, l'Algérie, mais qu'ils appartiennent bien à la France. Il s'agit de revendiquer, de manière pacifique et musicale, le fait d'appartenir à une France mondiale, comme celle racontée par Patrick Boucheron dans son Histoire mondiale de la France. «Une première solution à apporter pourrait être de construire une réflexion éditoriale, parce qu'il n'existe plus d'espace d'expression médiatique pour défendre ces musiques. En France, on ressent ce plafond de verre de l'establishment dont il est question dans le livre de Bertrand Dicale Ni noire ni blanche, histoires des musiques créoles. Je n'ai jamais ressenti aussi fortement l'exaspération des artistes qu'aujourd'hui. J'ai grandi dans des quartiers Sonacotra, où je ressentais l'exaspération des uns et des autres. On se tenait chaud en bas des tours d'immeubles. Mais aujourd'hui, me retrouvant en position d'acteur culturel dirigeant le festival Villes des musiques du Monde, qui m'expose et m'oblige, quelle réponse vais-je donner à l'exaspération de nos concitoyens d'ascendance haïtienne? La problématique de la "sono mondiale" rapportée à notre "pays mondial" est une matière compliquée... On appartient tous à des langues de France. "Le festival présente la "Cité des marmots". Pour cette opération, nous passons commande à des artistes pour qu'ils transmettent leur répertoire, leur patrimoine, à des enfants. La première édition a eu lieu en 2008 avec Habib Koité. À l'époque, le rectorat de Créteil avait réagi en me demandant pourquoi je voulais que des enfants de Seine-Saint-Denis apprennent le bambara. J'avais répondu que le bambara est la langue de France la plus parlée en SeineSaint-Denis... La problématique se trouve bien sur la question de la langue.

"Récemment, j'ai échangé avec l'une des cadres de la Délégation générale à la langue française et aux langues de France, service rattaché au ministère de la Culture. Je lui ai demandé si les quotas de musique à la radio ne pourraient pas être ouverts à toutes les langues de France. Elle m'a répondu qu'en effet, il existait peut-être là un espace juridique dans lequel pourraient s'engouffrer collectivement les défendeurs de musiques de clochers comme de musiques communautaires, pour revendiquer la défense des musiques régionales de notre monde nouveau, qui font la sono mondiale de 2018... L'esprit du Prix des Musiques d'ici réside dans la digestion de cette histoire collective.»

Les lauréats du Prix des Musiques d'ici en 2018 sont Walid Ben Selim, Germaine Kobo et Bella Lawson, et Edgar Sekloka. 